\title{
Asymmetric Expansion of the Youngest Galactic Supernova Remnant G1.9+0.3
}

\author{
Kazimierz J. Borkowski ${ }^{1}$, Peter Gwynne ${ }^{1}$, Stephen P. Reynolds ${ }^{1}$, David A. Green ${ }^{2}$, Una Hwang ${ }^{3}$, \\ Robert Petre $^{4}$, and Rebecca Willett ${ }^{5}$ \\ ${ }^{1}$ Department of Physics, North Carolina State University, Raleigh, NC 27695-8202, USA; kborkow@ncsu.edu \\ ${ }^{2}$ Cavendish Laboratory; 19 J.J. Thomson Ave., Cambridge CB3 0HE, UK \\ ${ }^{3}$ Department of Astronomy, University of Maryland, College Park, MD 20742, USA \\ ${ }^{4}$ NASA/GSFC, Code 660, Greenbelt, MD 20771, USA \\ ${ }^{5}$ Department of Electrical and Computing Engineering, University of Wisconsin-Madison, Madison, WI 53706, USA \\ Received 2017 January 11; revised 2017 February 8; accepted 2017 February 16; published 2017 March 1
}

\begin{abstract}
The youngest Galactic supernova remnant (SNR) G1.9+0.3, produced by a (probable) SN Ia that exploded $\sim 1900$ $\mathrm{CE}$, is strongly asymmetric at radio wavelengths, much brighter in the north, but bilaterally symmetric in X-rays. We present the results of X-ray expansion measurements that illuminate the origin of the radio asymmetry. We confirm the mean expansion rate (2011-2015) of $0.58 \% \mathrm{yr}^{-1}$, but large spatial variations are present. Using the nonparametric "Demons" method, we measure the velocity field throughout the entire SNR, finding that motions vary by a factor of 5 , from $0 . " 09$ to $0 . " 44 \mathrm{yr}^{-1}$. The slowest shocks are at the outer boundary of the bright northern radio rim, with velocities $v_{s}$ as low as $3600 \mathrm{~km} \mathrm{~s}^{-1}$ (for an assumed distance of $8.5 \mathrm{kpc}$ ), much less than $v_{s}=12,000-13,000 \mathrm{~km} \mathrm{~s}^{-1}$ along the X-ray-bright major axis. Such strong deceleration of the northern blast wave most likely arises from the collision of SN ejecta with a much denser than average ambient medium there. This asymmetric ambient medium naturally explains the radio asymmetry. In several locations, significant morphological changes and strongly nonradial motions are apparent. The spatially integrated X-ray flux continues to increase with time. Based on Chandra observations spanning $8.3 \mathrm{yr}$, we measure its increase at $1.3 \% \pm 0.8 \% \mathrm{yr}^{-1}$. The SN ejecta are likely colliding with the asymmetric circumstellar medium ejected by the SN progenitor prior to its explosion.
\end{abstract}

Key words: ISM: individual objects (G1.9+0.3) - ISM: supernova remnants - X-rays: ISM

Supporting material: data behind figure

\section{Introduction}

The youngest Galactic supernova remnant (SNR) G1.9+0.3 (Reynolds et al. 2008) has provided important new information on the very early development of an SNR as it interacts with the immediate SN environment. Its rapid expansion $\left(\sim 13,000 \mathrm{~km} \mathrm{~s}^{-1}\right.$, for a presumed distance of $8.5 \mathrm{kpc}$; Carlton et al. 2011, hereafter C11) allows for the study of expansion over relatively short time baselines. In C11, we compared observations in 2007 (Epoch I) and 2009 (Epoch II) in X-rays to obtain a mean expansion rate of $0.642 \% \pm 0.049 \% \mathrm{yr}^{-1}$, or a mean expansion age of $156 \pm 11$ yr. Assuming a mean deceleration rate $m\left(R \propto t^{m}\right)$ of about $m=0.7$, this implies a remnant age of $100 \mathrm{yr}$. However, the expansion is far from uniform. A much longer (1 Ms) observation in 2011 (Epoch III) allowed the detection of departures from uniformity in expansion along the major axis (Figure 1): the expansion varies systematically with radius, decreasing from $0.84 \% \pm 0.06 \% \mathrm{yr}^{-1}$ to $0.52 \% \pm 0.03 \% \mathrm{yr}^{-1} \quad$ (Borkowski et al. 2014, hereafter B14). This difference was ascribed either to asymmetry in the surrounding medium, or in the ejecta themselves, indicating a significantly anisotropic explosion. We have now obtained an additional observation in X-rays of $400 \mathrm{ks}$ (2015), which allows further study of nonhomologous expansion in G1.9+0.3. These observations should inform ongoing work on modeling G1.9+0.3 (e.g., Tsebrenko \& Soker 2015a, 2015b; Chakraborti et al. 2016; Yang et al. 2016).

The high expansion velocities, absence of an obvious pulsarwind nebula, and bilateral symmetry of the X-ray emission (as in SN 1006; Winkler et al. 2014) suggest that G1.9+0.3 originated in a Type Ia event. The detailed dynamics can then provide clues to the nature of these explosions, at an age when ejecta still dominate the dynamics. Thermal emission from $\mathrm{Si}$, $\mathrm{S}$, and $\mathrm{Fe}$ in isolated regions has allowed a picture to be drawn of highly anisotropic, overturned material (Borkowski et al. 2013).

One mystery, evident from the earliest X-ray observations, was the difference in morphology between radio and $\mathrm{X}$-ray images. The radio image (Figure 1) shows a broad maximum across the northern rim, rather than bilaterally symmetric rims in the southeast and northwest. Since all the emission is synchrotron, this morphological contrast is puzzling. SN 1006, for instance, has virtually identical images in radio and nonthermal X-rays (Winkler et al. 2014). The simplest explanation involves assuming that radio-bright but X-ray faint regions result from an energy distribution of relativistic electrons that is larger than that from X-ray bright regions at radio-emitting energies of order $1 \mathrm{GeV}$, but which cuts off before reaching X-ray emitting energies. One must then search for a mechanism to produce such differences.

\section{Observations}

\subsection{X-Ray}

The most recent (Epoch IV) Chandra observations of G1.9 +0.3 took place in the spring and summer of 2015, with 11 individual pointings (Table 1) with a combined effective exposure time of $392 \mathrm{ks}$, with the remnant again placed on the Advanced CCD Imaging Spectrometer (ACIS) S3 chip. In order to reduce particle background, Very Faint Mode was used. All observations were reprocessed with CIAO v4.7 and CALDB 4.6.7, then spatially aligned to the longest pointing 


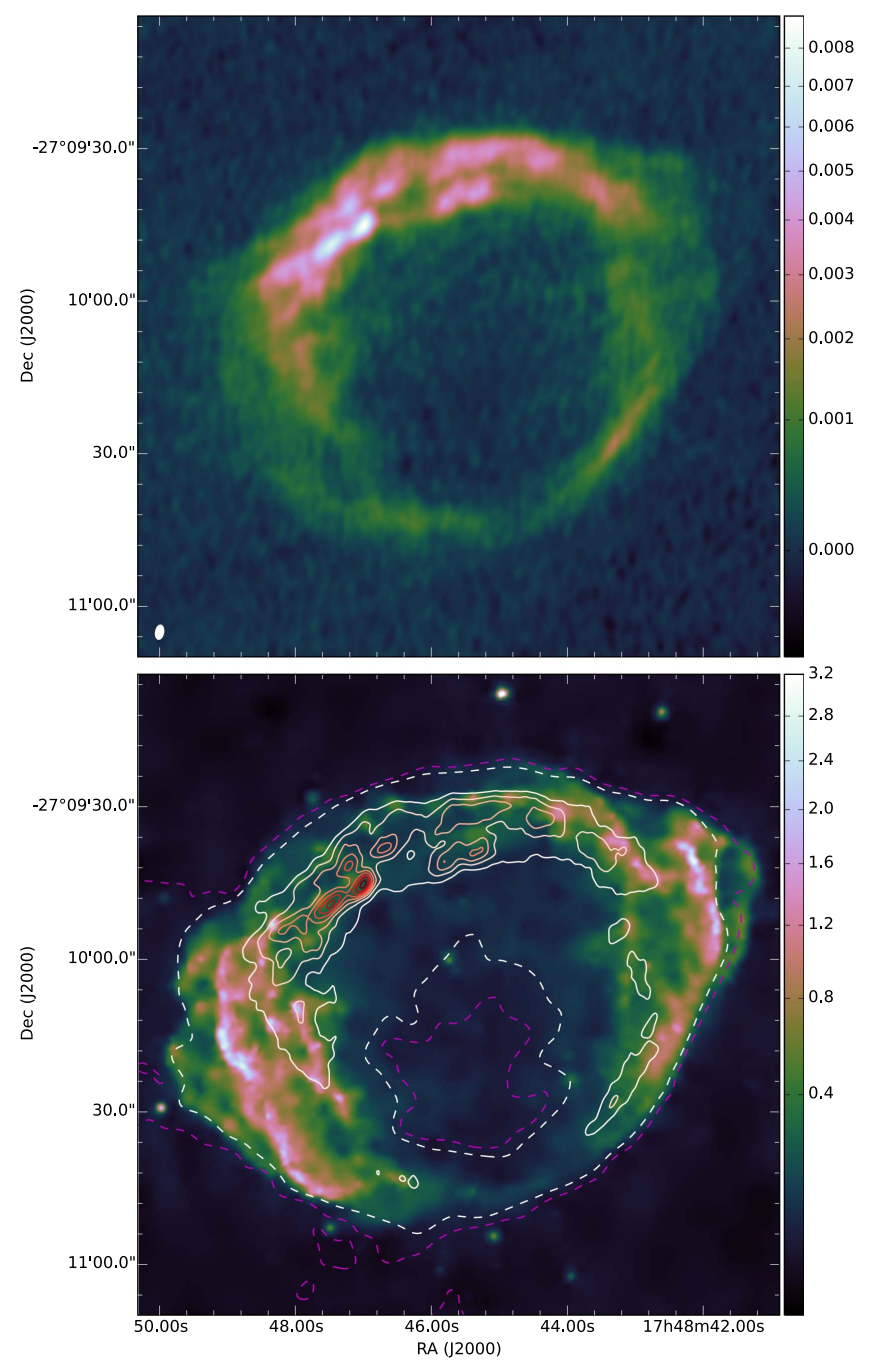

Figure 1. Top: Total intensity VLA image of G1.9+0.3 at $1365 \mathrm{MHz}$. The resolution is 2 !" $8 \times 1$." 6 at a PA of -9.6 (as shown in the left corner). The scale is in Jy beam ${ }^{-1}$. Bottom: Smoothed $20091.2-8 \mathrm{keV}$ Chandra image overlaid with selected radio contours emphasizing bright (solid lines from 1 to $8 \mathrm{mJy}_{\text {beam }}{ }^{-1}$ spaced by $1 \mathrm{mJy}^{\text {beam }}{ }^{-1}$ ) and very faint (dashed lines in magenta and white at 0.06 and $0.12 \mathrm{mJy}^{-1}$ beam $^{-1}$ ) emission. The scale is in counts per 0 ." $246 \times 0$ ". 246 image pixel (half an ACIS pixel). Intensities are shown with the cubehelix color scheme of Green (2011).

Table 1

Chandra Observations of G1.9+0.3 in 2015

\begin{tabular}{lccc}
\hline \hline Date & Observation ID & $\begin{array}{c}\text { Roll Angle } \\
(\mathrm{deg})\end{array}$ & $\begin{array}{c}\text { Effective Exposure Time } \\
(\mathrm{ks})\end{array}$ \\
\hline May 04-05 & 16947 & 87 & 39 \\
May 05-07 & 17651 & 87 & 111 \\
May 09-10 & 17652 & 87 & 26 \\
May 20 & 16949 & 75 & 9 \\
Jul 14-15 & 16948 & 272 & 40 \\
Jul 15 & 17702 & 272 & 37 \\
Jul 17 & 17699 & 272 & 20 \\
Jul 24 & 17663 & 272 & 57 \\
Jul 25 & 17705 & 272 & 10 \\
Aug 31 & 17700 & 260 & 29 \\
Sep 01-02 & 18354 & 260 & \\
\hline
\end{tabular}

from early May (Obs. ID 17651) using photons from the remnant itself (see $\mathrm{C} 11$ and $\mathrm{B} 14$ for a description of the alignment method). Numerous point sources present near G1.9
+0.3 were then used to perform the final alignment to the deep (977 ks) Epoch III observation. The exposure-weighted time interval $\Delta t$ between Epochs III and IV is $4.057 \mathrm{yr}$. While shorter $49.6 \mathrm{ks}$ Epoch I and $237 \mathrm{ks}$ Epoch II observations provide longer time baselines $(8.331 \mathrm{yr}$ and $5.918 \mathrm{yr}$, respectively), this advantage is more than offset by the increased counting noise compared to the long Epoch III observations. Therefore, the expansion measurements described here are based on the Epoch III and IV observations alone.

Images, $512^{2}$ in size, were extracted from the merged event files by binning event positions to half the ACIS pixel size, so one image pixel is 0 !! $246 \times 0$ !! 246 . We also extracted data cubes, $512^{2} \times 15$ in size, using the same spatial pixel size, while spectral channels from 76 to 555 (1-8 keV energy range) were binned by a factor of 32 .

X-ray spectra were extracted from individual rather than merged event files, and then summed (response files were averaged). Spectral analysis was performed with XSPEC v12.8.1 (Arnaud 1996), using C-statistics (Cash 1979). The background was modeled rather than subtracted. Spectra of G1.9+0.3 were modeled with an absorbed power law, using the solar abundances of Grevesse \& Sauval (1998) in the phabs absorption model.

\subsection{Radio}

G1.9+0.3 was observed with the Karl G. Jansky Very Large Array (VLA) in three configurations between 2008 December and 2009 July, in the $L$-band. Two observations were made in the A array, each $5.7 \mathrm{hr}$ on source, and one each in the B and C arrays, each $0.6 \mathrm{hr}$ on source. The observations were made with two $25 \mathrm{MHz}$ bandwidths. However, one of these was badly affected by interference, so the results presented here are from a single $25 \mathrm{MHz}$ bandwidth, centered at $1365 \mathrm{MHz}$. The observations were calibrated using standard techniques in the Astronomical Image Processing System, and combined for imaging. Figure 1 shows the image of G1.9+0.3, with a resolution of 2 !" $8 \times 1$ !" 6 at a PA of -9.6 , which has an r.m.s. noise of $\approx 0.054 \mathrm{mJy}^{\text {beam }}{ }^{-1}$. The inclusion of the smaller configurations in these observations, i.e., the $\mathrm{B}$ and $\mathrm{C}$ arrays, means that this image is sensitive to structures on all scales from G1.9+0.3. Radio contours, overlaid over a smoothed 2009 Chandra image in Figure 1, show that the bright radio emission in the north is bounded on the outside by faint X-ray emission that marks the location of the primary blast wave there. Similarly, radio emission in the southeast and northwest is also bounded on the outside by X-ray filaments, but this time $\mathrm{X}$-rays are bright and the radio emission is faint. Radio contours at low surface brightness (Figure 1) show that this contrast between bright $\mathrm{X}$-rays and faint radio is most conspicuous for the outermost protrusions ("ears") in the southeast and northwest.

\section{Expansion}

Relatively simple, parametric methods for measuring expansion (such as those used in $\mathrm{C} 11$ and B14) cannot describe the very complex expansion of $\mathrm{G} 1.9+0.3$ revealed by the new Chandra observations. We now use the "Demons" algorithm of Thirion (1998), as implemented in the SimpleITK software package (Lowekamp et al. 2013), to measure the nonuniform expansion of G1.9+0.3. Attributing morphological changes only to motions, the Demons method provides a 
nonparametric way for measuring these motions globally. Since this computationally efficient method (approximately) minimizes the sum of squared differences between pixel intensities, we need to apply it to the smoothed images, not to raw Chandra images that are badly affected by counting noise.

We smoothed Chandra data with the non-local PCA method of Salmon et al. (2014), with smoothed images extracted from smoothed data cubes. This method combines elements of dictionary learning and sparse patch-based representation of images (or spectral data cubes) for photon-limited data. Because this Poisson-PCA method is computationally intensive, relatively small $\left(512^{2} \times 15\right)$ data cubes, heavily binned along the spectral dimension as described above, were smoothed using patches $10^{2} \times 6$ in size. The moderate spatial patch size of 2 " $46 \times 2$ ". 46 preserves sharp spatial structures seen in the bright filamentary features, while a large patch size in the spectral dimension is suitable for the synchrotrondominated spectra of G1.9+0.3 that vary smoothly across the entire spectral range of Chandra. With the patch size chosen, the most important parameters that control the smoothing of data cubes are the rank $l$ of the approximation to the underlying intensity to the collection of patches (order of the Poisson-PCA method), and the number of clusters $K$ into which patches are grouped prior to estimation of intensities. We used $l=6$ and $K=30$ for the 2011 and 2015 data cubes of $\mathrm{G} 1.9+0.3$.

The expansion of G1.9+0.3 is readily apparent by comparing smoothed Chandra images from 2011 and 2015 (Figure 2). We measure an average expansion rate of $0.58 \% \mathrm{yr}^{-1}$ between these two epochs (using the same method as in C11), but there are very large deviations from uniform expansion. In only four years, striking morphological variations at various locations within the remnant can be discerned by eye. These include bending of the innermost rim in the west, and even more complex, strongly nonradial motions in the northeast where changes in relative positions of prominent emission knots and filaments can be seen. In the framework of the Demons algorithm, which can account for such complex motions, the $20111-8 \mathrm{keV}$ smoothed image is considered as the reference image, while the $20151-8 \mathrm{keV}$ smoothed image is the moving image. Before application of the Demons method, a particle background with an estimated rate of $9.0 \times 10^{-5}$ and $7.0 \times 10^{-5}$ cts s $^{-1}$ per image pixel was subtracted from the 2011 and 2015 images, respectively, and then each background-subtracted image was normalized using a corresponding monochromatic ( $E=3 \mathrm{keV}$ ) exposure map. Point sources and the featureless interior of the remnant have been masked out. The uniform expansion at $0.58 \% \mathrm{yr}^{-1}$ centered 10 .' 5 north of the geometrical center was used to set initial displacements between the two epochs, then at each iteration step of the Demons method computed displacements were smoothed with a Gaussian with FWHM of 0."82. ${ }^{6}$ After achieving convergence, this smoothing resulted in a good match between the 2011 image and the (transformed) 2015 image (Figure 3), demonstrating the effectiveness of the Demons method, but the final displacement vectors were affected by substantial noise. We reduced this noise by calculating brightness-weighted averages of displacement vectors within various regions of interest. Finally, these averaged displacements were divided by $\Delta t$ to obtain proper motion vectors $\boldsymbol{\mu}$.

\footnotetext{
6 Other parameters of the Demons method are far less important and primarily affect its convergence rate.
}

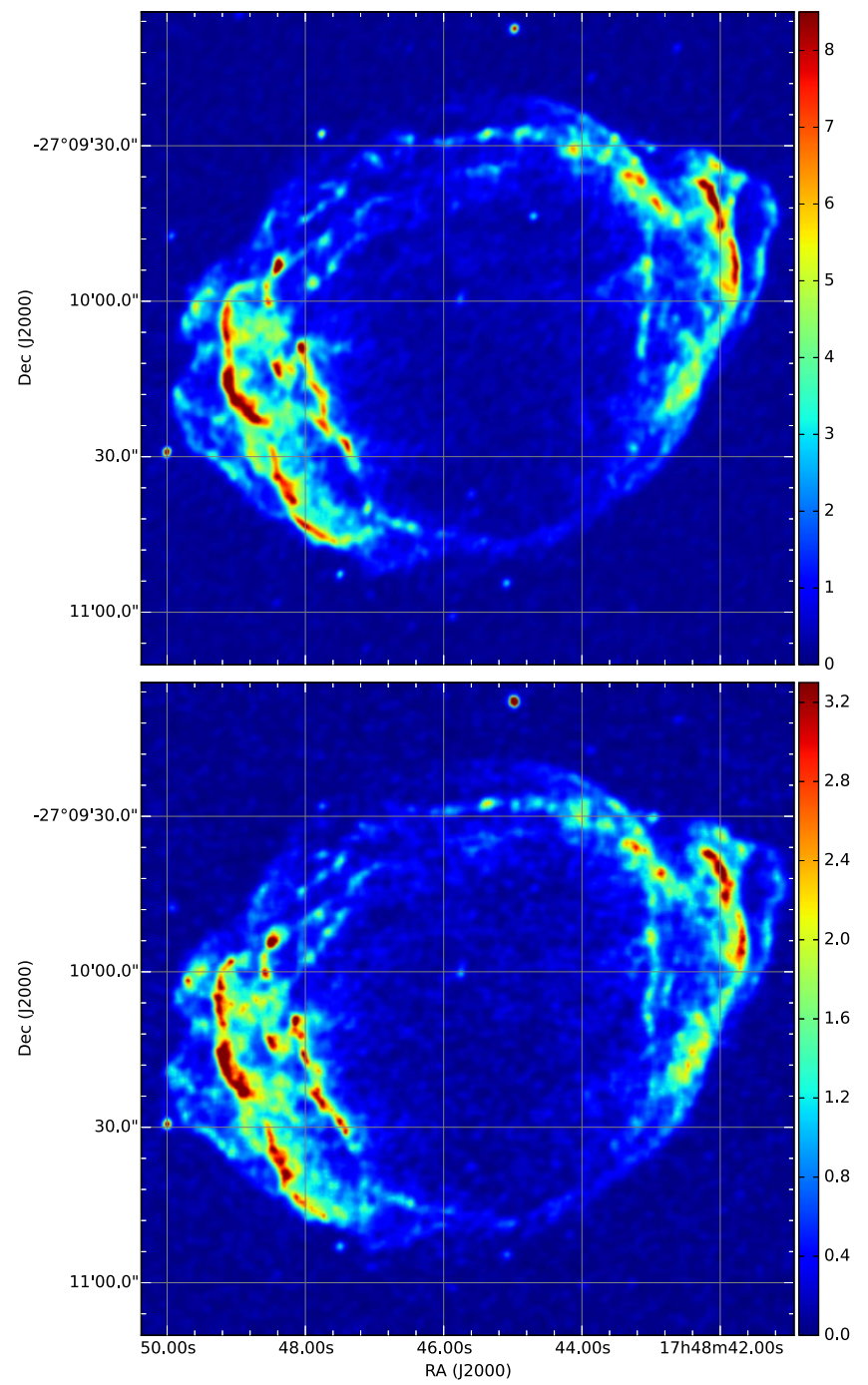

Figure 2. Smoothed X-ray images of $\mathrm{G} 1.9+0.3$ in the $1-8 \mathrm{keV}$ energy range from 2011 (top) and 2015 (bottom), with a coordinate grid superposed. Expansion between these Epoch III and IV observations is apparent. The scale is in counts per 0 !" $246 \times 0$ !' 246 image pixel.

We show vectors $\boldsymbol{\mu}$ in Figure 3 for a large number of regions chosen to delineate all major morphological features of G1.9 +0.3 . The white arrow with length 0 .' $25 \mathrm{yr}^{-1}\left(10,100 \mathrm{~km} \mathrm{~s}^{-1}\right.$ at $8.5 \mathrm{kpc}$ ) shows their scale, while their tails are located at the geometrical centroids of these regions. Motions are between 0 " $09 \mathrm{yr}^{-1}$ and 0 ". $44 \mathrm{yr}^{-1}$, varying by a factor of up to 5 . The median $\mu$ is 0 !' $28 \mathrm{yr}^{-1}$. The slowest shocks are in the north. The fastest motions are predominantly along the major axis of the remnant. Motions are strongly nonradial with respect to the remnant's geometrical center at R.A. $17^{\mathrm{h}} 48^{\mathrm{m}} 45^{\mathrm{s}} \cdot 61$, decl. $-27^{\circ}$ $10^{\prime} 05$ !' 6 . We infer the explosion occurred somewhere northeast of the geometrical center. Extreme deviations from radial motions are present.

In Figure 4, we draw vectors $\boldsymbol{\mu}$ over the radio and contemporaneous 2009 Chandra images (tails of vectors were shifted inward to account for the remnant's expansion between the 2008 December array A VLA and Epoch III Chandra observations). Expansion is noticeably slower along the outer boundary of the radio emission. The median motion along the outer radio contour at $1.5 \mathrm{mJy} \mathrm{beam}^{-1}$ (regions $a$ to $n$ in Figure 4) is $0 . \prime 17 \mathrm{yr}^{-1}$ (only $6900 \mathrm{~km} \mathrm{~s}^{-1}$ ), significantly less 

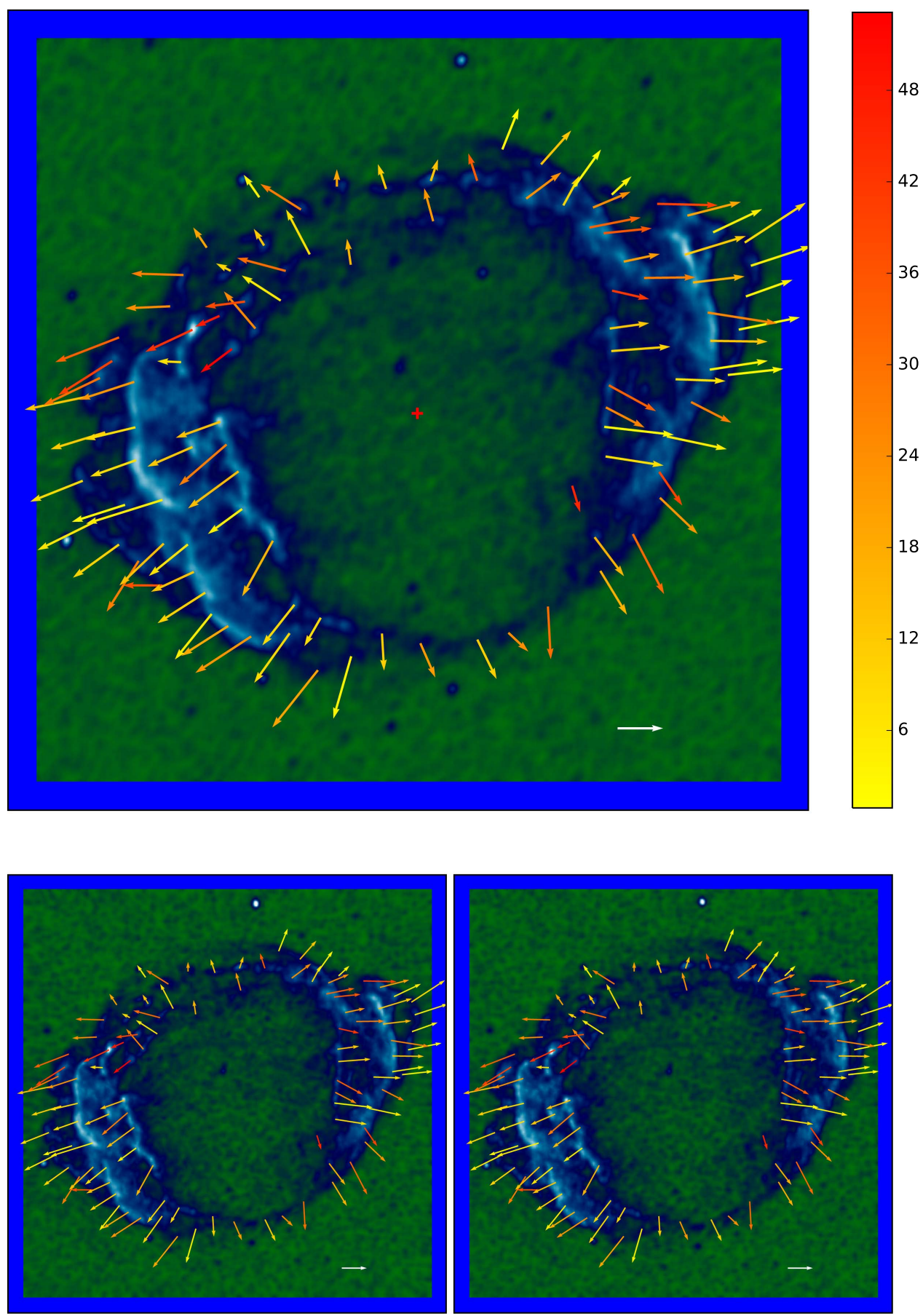

Figure 3. Proper motion vectors overlaid on the 2011 (top), 2015 (bottom right), and the transformed 2015 (bottom left) images. They are color-coded according to the deviations in direction from radial (with respect to the geometrical center of the remnant, marked by the red cross), in degrees according to the vertical scale. The white arrows indicate $0 . " 25 \mathrm{yr}^{-1}$.

(The data used to create this figure are available)

than the overall median of $0.28 \mathrm{yr}^{-1}\left(11,000 \mathrm{~km} \mathrm{~s}^{-1}\right)$. Among all regions with $\mu<0$ ". $17 \mathrm{yr}^{-1}$, most (12 out of 14) are within the northern rim, predominantly toward the north and northeast where radio emission is particularly bright. This includes regions $f \quad\left(0\right.$ !' $\left.16 \mathrm{yr}^{-1}\right), \quad g \quad\left(0\right.$ !' $\left.13 \mathrm{yr}^{-1}\right), \quad h \quad\left(0{ }^{\prime \prime} 14 \mathrm{yr}^{-1}\right)$, $i \quad\left(00^{\prime \prime} 095 \mathrm{yr}^{-1}\right), \quad k \quad\left(0{ }^{\prime \prime} 11 \mathrm{yr}^{-1}\right), \quad l \quad\left(0{ }^{\prime \prime} 089 \mathrm{yr}^{-1}\right), \quad$ and $n$ $\left(0\right.$ "' $\left.13 \mathrm{yr}^{-1}\right)$. The smallest $\mu$ (region $l$ ) corresponds to a velocity of only $3600 \mathrm{~km} \mathrm{~s}^{-1}$. But motions of several knots and filaments in the northeast are larger, more typical for the remnant as a whole, including regions $j\left(0,26 \mathrm{yr}^{-1}\right)$ and $m$ $\left(0^{\prime \prime} 29 \mathrm{yr}^{-1}\right)$. The latter corresponds to a very bright X-ray knot with motion strongly deviating from radial (by $42^{\circ}$ ), while the former is the northernmost of five fast-moving knots/filaments that are mostly located near where G1.9+0.3 is the brightest in radio.

The motions along the northwest outer radio contour (regions $a-e)$ are $0 . \prime 20 \mathrm{yr}^{-1}, 0 . \prime 27 \mathrm{yr}^{-1}, 0 ! 29 \mathrm{yr}^{-1}, 00^{\prime \prime} 19 \mathrm{yr}^{-1}$, and $0 ! 24 \mathrm{yr}^{-1}$, respectively, with a median of $0.24 \mathrm{yr}^{-1}$ $\left(9700 \mathrm{~km} \mathrm{~s}^{-1}\right)$. This is significantly more than those in the north-northeast, where the median for regions $f-n$ is 

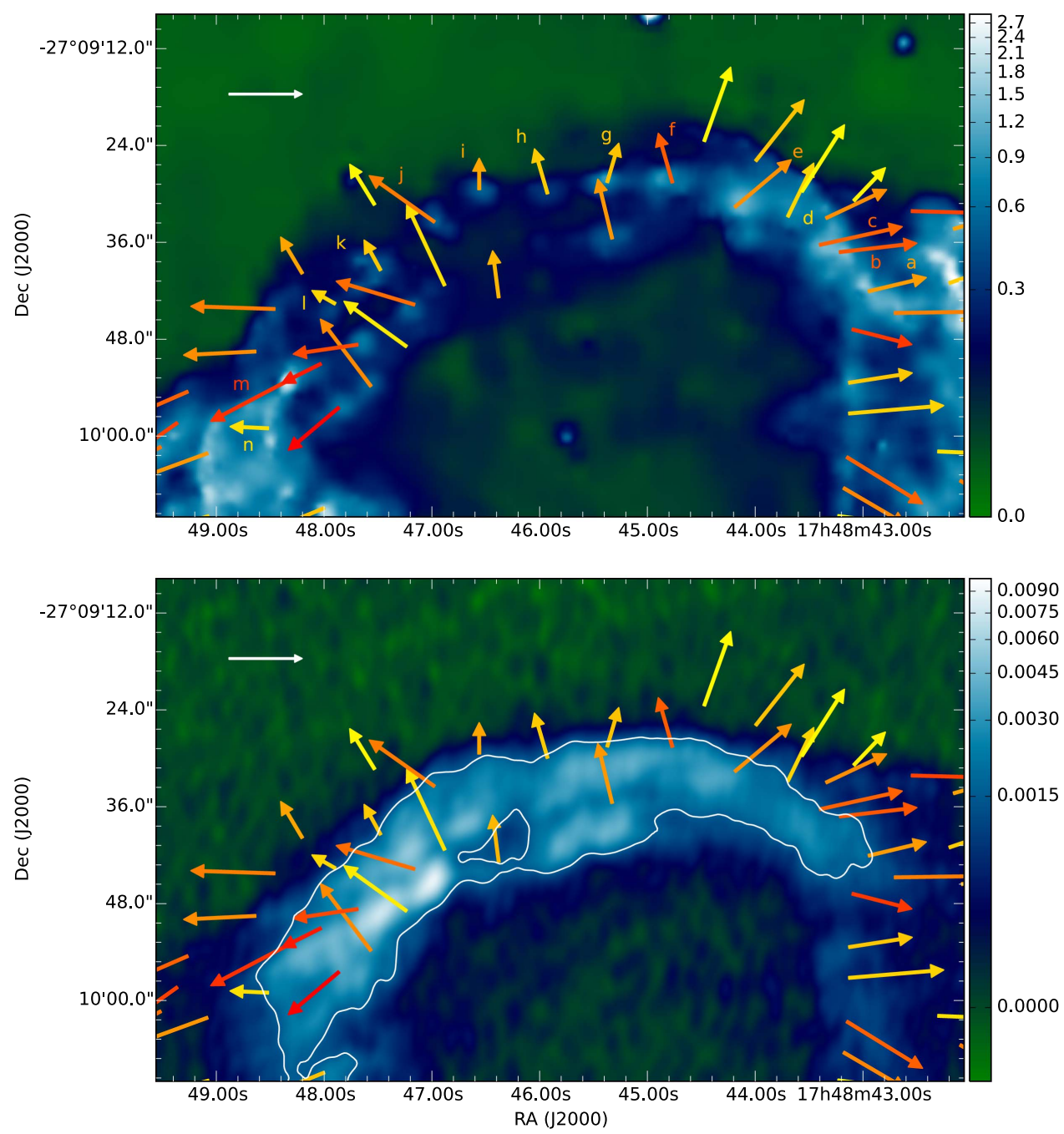

Figure 4. 2009 X-ray (top) and radio (bottom) images centered on the radio-bright northern rim, with X-ray proper motion vectors overlaid (color-coded as in Figure 3). The white arrows indicate 0 "' $25 \mathrm{yr}^{-1}$. The radio contour is at $1.5 \mathrm{mJy}$ beam ${ }^{-1}$. Selected vectors along this contour have been labeled. The scales are in the same units as in Figure 1. Note the very low velocities just ahead of the outermost bright radio emission.

0 "' $13 \mathrm{yr}^{-1}\left(5300 \mathrm{~km} \mathrm{~s}^{-1}\right)$, but still less than average for G1.9 +0.3 . As in the northeast, strongly nonradial motions are present. Unlike the north-northeast where X-rays are generally faint and radio is quite bright, this region is moderately bright both in $\mathrm{X}$-rays and radio.

The motions along the major axis are faster than the overall median of $0 . \prime 28 \mathrm{yr}^{-1}$. For the brightest X-ray rims in the middle, the median is $0.132 \mathrm{yr}^{-1}$, increasing modestly to 0 " $33 \mathrm{yr}^{-1}$ ahead of these rims. For the innermost rims (this includes vectors $\boldsymbol{\mu}$ within the inner pair of regions in B14), the median drops to $0.130 \mathrm{yr}^{-1}$. These rather modest $(12,000-$ $13,000 \mathrm{~km} \mathrm{~s}^{-1}$ ) radial variations along the major axis are not surprising in view of the rapid radial decrease in expansion rates found by $\mathrm{B} 14$.

For the southern radio rim, the motion is $0 . / 21 \mathrm{yr}^{-1}$ (the median for 7 regions along this rim). This is significantly more than the 0 . $^{\prime} 13 \mathrm{yr}^{-1}$ that we found in the north-northeast, and corresponds to velocity of $8300 \mathrm{~km} \mathrm{~s}^{-1}$. The southernmost extension toward the east, with only a very faint radio counterpart, must be moving much faster than this, as we find $\mu=0$.' $38 \mathrm{yr}^{-1}\left(15,000 \mathrm{~km} \mathrm{~s}^{-1}\right)$. Apparently, the motions are also complex in this relatively faint region of the remnant.

\section{Flux Increase}

A joint fit to the spatially integrated $1-9 \mathrm{keV}$ spectrum of G1.9+0.3 and to the background spectrum gave a best-fit photon index $\Gamma$ of $2.45 \pm 0.06$ and an absorbing column density $N_{\mathrm{H}}=7.41( \pm 0.16) \times 10^{22} \mathrm{~cm}^{-2}$, with an absorbed $1-7 \mathrm{keV}$ flux $F_{2015}$ of $3.063( \pm 0.027) \times 10^{-12} \mathrm{erg} \mathrm{cm}^{-2} \mathrm{~s}^{-1}$ (errors are $90 \%$ confidence intervals). Both $\Gamma$ and $N_{\mathrm{H}}$ are consistent with previous measurements $(\Gamma=2.40 \pm 0.03$ and $\left.N_{\mathrm{H}}=7.25( \pm 0.09) \times 10^{22} \mathrm{~cm}^{-2} ; \mathrm{B} 14\right)$, while the flux increased by $3 \%$ since 2011. So G1.9+0.3 continues to increase in brightness, but at a somewhat lower rate than the $1.9 \% \mathrm{yr}^{-1}$ reported in $\mathrm{B} 14$.

A linear regression fit to $F_{2015}$ and to the previously

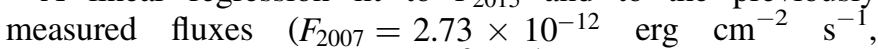
$F_{2009}=2.88 \times 10^{-12} \mathrm{erg} \mathrm{cm} \mathrm{cm}^{-2} \mathrm{~s}^{-1}, \quad F_{2011}=2.97 \times 10^{-12}$ erg $\mathrm{cm}^{-2} \mathrm{~s}^{-1}$; B14) gives a rate of $1.3 \% \pm 0.8 \% \mathrm{yr}^{-1}$. Flux residuals (model fit minus observations) range from $-1.4 \%$ (2011) to $1.2 \%$ (2007), comparable to the $(1 \sigma)$ statistical error of $1.3 \%$ for $F_{2007}$ but more than the $0.3 \%-0.6 \%$ found at other epochs. Although only about 8000 source counts were detected in the short 2007 observation, systematic errors are already becoming comparable to statistical errors for Chandra spectra 
with that number of counts $\left(\sim 10^{4}\right)$. The much longer observations from later epochs are then dominated by systematic (not statistical) errors, and the errors are likely comparable for all four epochs. In this case, the flux residuals quoted above are consistent with a linear flux increase from 2007 to 2015. Our newly determined, more accurate rate of $1.3 \% \mathrm{yr}^{-1}$, with the relatively large error of $\pm 0.8 \% \mathrm{yr}^{-1}$ accounting for systematic effects, is also consistent with the larger rate of $1.9 \% \mathrm{yr}^{-1}\left( \pm 0.4 \% \mathrm{yr}^{-1}\right.$ — statistical errors only) based on the Epoch I-III observations alone (B14).

\section{Discussion}

Figure 4 shows that the slowest expansion speeds are found just beyond the radio maxima. Evidently the shock is encountering denser material there, resulting in a drastic deceleration of the expansion. Such dense material in a slower shock will have two effects on the accelerated electron spectrum: a larger number density of accelerated electrons (since that density is generally found to be a fraction $\sim 10^{-4}$ of the total particle density; e.g., Ellison \& Cassam-Chenaï 2005), and a lower maximum energy, since $E_{\max } \propto B v_{\text {shock }}^{2}$ for agelimited acceleration (e.g., Reynolds 2008). The resulting synchrotron emission will be brighter in radio than that from surrounding regions with faster shocks, but the spectrum will turn over at lower energies and may not reach to X-rays. That is, the slower shocks we observe, produced by denser ambient material, can explain the differing radio and X-ray morphologies. Assume (conservatively) $B \propto \rho^{1 / 2}$ (no extra magneticfield amplification). Then, since we expect $\rho v_{\text {shock }}^{2} \sim$ const., we have $E_{\max }$ (age) $\propto \rho^{-1 / 2} \propto v_{\text {shock }}$, and $\nu_{\text {roll }} \propto E_{\text {max }}^{2} B \propto v_{\text {shock }}$. In the region of the radio maximum, the mean shock speed is about $4000 \mathrm{~km} \mathrm{~s}^{-1}$, or about 0.3 of the value at the bright rims. The integrated spectrum of G1.9+0.3 is well described by a rolloff of $3.1 \times 10^{17} \mathrm{~Hz}$ (Zoglauer et al. 2015), implying a rolloff near the radio peak of about $9 \times 10^{16} \mathrm{~Hz}$ or less than 0.4 $\mathrm{keV}$ - too low to produce appreciable X-ray synchrotron emission at that location.

The rate of flux increase we find, $1.3 \% \mathrm{yr}^{-1}$, is still in the range of radio increase rates of $1 \%-2 \% \mathrm{yr}^{-1}$ (Green et al. 2008; Murphy et al. 2008), so the important question of the comparison of radio and X-ray rates is still unsettled. Different rates imply time-variation in the maximum electron energies, or something even more unexpected. Further observations of both radio and X-rays will be crucial.
The large range of velocity vectors, in both magnitude and direction, that we find in G1.9+0.3 highlights the inadequacy of spherically symmetric models for describing SN events and their aftermath. The north rim is particularly poorly described by $1 \mathrm{D}$ models. Failure to account for the large variations in velocity casts doubt on such analyses. However, these velocities contain a great deal of information on the explosion and the circumstellar environment that rivals Kepler's SN, the most recent historical SN in the Galaxy, in its complexity. As in Kepler's Type Ia SNR, large $(>10)$ density gradients are present, and SN ejecta are likely colliding with the asymmetric circumstellar medium ejected by the SN progenitor. Our statistical method of determining velocities throughout an expanding remnant, and not just in well-defined sharp outer edges, should have wide application in following the expansion of young SNRs and elucidating their early evolution and the nature of the surrounding medium.

We acknowledge support by NASA through Chandra General Observer Program grants SAO GO5-16069A-C.

Facilities: $C X O$, VLA.

\section{References}

Arnaud, K. A. 1996, in ASP Conf. Ser. 101, Astronomical Data Analysis and Systems V, ed. G. Jacoby \& J. Barnes (San Francisco, CA: ASP), 17

Borkowski, K. J., Reynolds, S. P., Green, D. A., et al. 2014, ApJL, 790, L18 Borkowski, K. J., Reynolds, S. P., Hwang, U., et al. 2013, ApJL, 771, L9 Carlton, A. K., Borkowski, K. J., Reynolds, S. P., et al. 2011, ApJL, 737, L22 Cash, W. 1979, ApJ, 228, 939

Chakraborti, S., Childs, F., \& Soderberg, A. 2016, ApJ, 819, 37

Ellison, D. C., \& Cassam-Chenaï, G. 2005, ApJ, 632, 920

Green, D. A. 2011, BASI, 39, 289

Green, D. A., Reynolds, S. P., Borkowski, K. J., et al. 2008, MNRAS, 387, L54

Grevesse, N., \& Sauval, A. J. 1998, SSRv, 85, 161

Lowekamp, B. C., Chen, D. T., Ibáñez, L., \& Blezek, D. 2013, Front. Neuroinform., 7, 45

Murphy, T., Gaensler, B. M., \& Chatterjee, S. 2008, MNRAS, 389, L23

Reynolds, S. P. 2008, ARA\&A, 46, 89

Reynolds, S. P., Borkowski, K. J., Green, D. A., et al. 2008, ApJL, 680, L41

Salmon, J., Harmany, Z., Deledalle, C.-A., \& Willett, R. 2014, J. Math. Imaging Vis., 48, 279

Thirion, J.-P. 1998, Med. Image Anal., 2, 243

Tsebrenko, D., \& Soker, N. 2015a, MNRAS, 450, 1399

Tsebrenko, D., \& Soker, N. 2015b, MNRAS, 453, 166

Winkler, P. F., Williams, B. J., Reynolds, S. P., et al. 2014, ApJ, 781, 65

Yang, R., Sun, X., \& Aharonian, F. 2016, A\&A, submitted (arXiv:1612.02262)

Zoglauer, A., Reynolds, S. P., An, H., et al. 2015, ApJ, 798, 98 